\title{
Crow Instability Effects on the Exhaust Plume Mixing and Condensation
}

\author{
S. Brunet, F. Garnier, P. Sagaut \\ Office National d'Etudes et de Recherches Aérospatiales, \\ 29, avenue de la Division Leclerc, 92320 Châtillon, France, \\ E-mail: sbrunet@onera.fr \\ European Series in Applied and Industrial Mathematics
}

\begin{abstract}
Three-dimensional direct numerical simulation is performed to study the interaction of two engine jets with a pair of trailing vortices. First of all, a test panel of selected flux limiter has permitted to suppress spurious oscillations of the passive scalar and to enforce its positivity. Then, contours of a passive scalar (or a tracer concentration) are used to investigate the mixing and entrainment processes when the vortex pair goes through sinusoidal instability. The present results show that the dilution of the exhaust plume is less efficient when the flow undergoes this perturbation. However, the rate of mixing appears insensitive to the development of the Crow instability. Furthermore, in order to study condensation process, results are presented involving the calculation of the saturation with respect to liquid water. This is performed by post-processing solution fields which provide a qualitative indicator where the condensation occurred in the aircraft wake.
\end{abstract}

\section{Introduction}

The future build-up of a large fleet of commercial aircraft, subsonic and supersonic, may provide a major atmospheric perturbation for the next decade. One critical point of the impact of aircraft emissions upon the atmosphere concerns the effect of aircraft exhaust on aerosol and cloud formation. These exhaust emissions contain mainly water vapor, carbon oxides, soots, unburned hydrocarbons and minor gases, due to the high combustion efficiency. For favorable ambient relative humidity and temperature, typically colder than $-40^{\circ} \mathrm{C}$, wing-tip vortices interact with ambient medium and exhaust plume to form condensation trails (or "contrails").

Even if the direct effects of the water vapor emissions seem negligible, the role in the Earth's radiative budget of the droplets and of the particles emitted by the jet engine remains unclear and needs more studies (Fortuin et al, 1995, Ponater et al, 1996). Recent conclusions (French Academy of Sciences, 1997) pointed out the great uncertainty concerning the role of contrails on cloud formation created by aircraft at the local scale, i.e. at the vortex wake scale. This mainly includes the knowledge of the mixing and condensation processes in the trailing vortices.

In cruise condition, the aircraft wake is composed of two counter-rotating wing-tip vortices. During the first seconds, the vorticity distribution from the wing rolls up into a pair of trailing vortices. These vortices do not evolve and decay by simple diffusion. Atmospheric turbulence and stratification enter in the stability of the two wing-tip vortices in the form of a non-linear forcing term (Crow and Bate, 1975) and as a consequence, the vortex break-up can appear, typically within a few minutes. Before vortex break-up, the wake dynamics is dominated by the interaction of the vortex pair through a mutual induction sinusoidal instability (so called the Crow instability).

The details of the mixing between the engine jets and vortex wake are complicated and involve several distinct processes. It is useful to define three regions. Firstly, the near field jet region is characterized by physical processes of usually co-flowing jets. Secondly the deflection region corresponds to the entrapment of the jet engine towards the vortex core and finally the shearing region where the jet streamlines present distortion and stretching generated by the rotational component of the velocity inside the vortex cores. For modern large transport aircraft, these three regions describe the jet evolution over the time periods of 
approximately, $0-4 \mathrm{~s}, 4-14 \mathrm{~s}$ and $14-50 \mathrm{~s}$ after the release of the hot jet from the nozzle exit into the atmosphere.

The effect of trailing vortices on the dilution and chemistry of the exhaust jets has been studied by several authors (Anderson et al, 1996, Kärcher et al, 1995, Garnier et al, 1997a). However, the process of contrail formation remains unclear. One of the causes is the lack of knowledge of accurate partial vapor pressure of water and temperature in the vortex wake.

This work's purpose is to investigate the influence of the Crow instability on the mixing and condensation processes which occur a few seconds to approximately one minute after the formation of the aircraft wake, but before vortex break-up. The initial configuration and properties of the exhaust jets which are important to the contrail formation, are taken from the computation of Garnier et al, (1997b).

\section{Model review}

\subsection{Computational model}

The mass and momentum equations are

$$
\begin{gathered}
\frac{\partial \rho}{\partial t}+\frac{\partial \rho u_{j}}{\partial x_{j}}=0 \\
\frac{\partial \rho u_{j}}{\partial t}+\frac{\partial}{\partial x_{j}}\left[\rho u_{i} u_{j}+p \delta_{i j}-\tau_{j i}\right]=0
\end{gathered}
$$

where $\tau_{j i}$ is the viscous stress tensor. With the law for perfect gas, the pressure reads

$$
p=\rho R T
$$

where $\mathrm{R}$ is the gas constant and $\mathrm{T}$ is the temperature. The total energy equation is

$$
\frac{\partial \rho E}{\partial t}+\frac{\partial}{\partial x_{j}}\left[(\rho E+p) u_{j}-u_{i} \tau_{j i}+q_{j}\right]=0
$$

where

$$
E=e+\frac{1}{2} u_{i} u_{i}
$$

and $q_{j}$ is the heat flux vector given by the Fourier law. We assume a constant Prandtl number of 0.7.

For the convective terms a sixth order compact finite difference scheme (Lele, 1990) is used and for the diffusion terms a second order accurate scheme is used in the all three directions. The time marching is performed using a low-storage Runge-Kutta third-order method (Lowery and Reynolds, 1986).

To assess the time evolution of the tracer concentration, an equation for a passive scalar $Z$ is solved simultaneously:

$$
\frac{\partial \rho Z}{\partial t}+\frac{\partial}{\partial x_{j}}\left[\rho Z u_{j}-\mu \frac{\partial Z}{\partial x_{j}}\right]=0 .
$$

In first approach, we consider the temperature and the density fields as constant. Thus, the coefficient of viscosity is constant in the following simulations.

Furthermore, a quantitative analysis of the mixing is given in terms of "mixedness" (Cetegen and Mohamad, 1993) which is defined by the following relation:

$$
f=\frac{1}{\Sigma} \int Z(1-Z) d s
$$

where $\Sigma$ represents the volume of the domain. This parameter measures the degree of mixing, formulated in term of second moment of the concentration field. It has a finite value since the far field of the vortex wake is surrounded by normalized concentration at $Z=0$. in a given domain, the value of $f$ varies between 0 for completely unmixed region (i.e. ambient air) to a finite value for scalar mixing. 


\subsection{Second order TVD scheme}

The main problem in fluid-flow simulation is the bounded discretization of convection terms in steep gradient zones. To achieve this goal, two methods are currently used: Flux-Corrected-Transport (FCT) (Boris and Book, 1976) and Flux-Limiters (FL). Practice shows that FCT schemes are demanding in computer time whereas FL schemes are less expensive. Higher order upwind linear convection schemes, although more stable than pure central differencing, still remain vulnerable to non-physical oscillations and do not maintain the positivity of the scalar. According to Godunov theorem no linear convection schemes of second order accuracy or higher can be monotonic. One of the solutions is the use of non-linear discretizations such as TVD schemes (Harten, 1983), which adjust themselves according to the local solution so as to maintain bounded behavior.

Waterson and Deconinck (1995) have tested and reviewed a set of existing non-linear schemes. Among the different limiters proposed we have been interested by six of them: Koren, Minmod, Smart, Superbee, van Albada and van Leer. General comparisons where described in Waterson's article using several standard incompressible viscous test cases. However attention here will be restricted to the convection of the scalar fields: Gaussian distribution case and Two-level step function. For this analysis, the velocity fields are assumed to take the following forms, in cylindrical coordinates, such as the solid body rotation:

$$
v_{\theta}=2 \pi r
$$

or the tangential velocity of the Lamb-Oseen vortex given by

$$
v_{\theta}=\frac{2 \pi}{r}\left(1-e^{-r^{2}}\right) .
$$

Thus among this wide variety of FL behavior we can hope to find the best convection scheme.

All the tests have been performed at infinite Peclet number on a 2D uniform grid (101×101 points) without taking into account the diffusion terms.

The time integration is by a second order Runge-Kutta scheme. For the whole result CFL $=0.125$.

The Table 1 sums up the computational test results. $Z_{\min }$ and $Z_{\max }$ represent the minimum and the maximum values of the passive scalar field, which are equals to 0 and 1; respectively. The root-mean square is given by the classical relation:

$$
R M S=\sqrt{\frac{1}{j_{\max }-1} \sum_{j=1}^{j_{\max }}\left(Z_{j}-Z_{j}^{\text {exact }}\right)^{2}} .
$$

It represents an assessment of the error between exact and numerical solutions after 1000 cycles. Note that 1000 cycles represent 1 revolution of the 2D distribution of passive scalar. Unfortunately, the RMS for the convection of the Gaussian scalar with the Lamb-Oseen velocity can not be calculated directly, and so there is not exact solution for comparison to the numerical results. 


\begin{tabular}{|l||l|l|l|l|}
\hline Test case & Flux Limiter & $Z_{\min }(0)$ & $Z_{\max }(1)$ & RMS error \\
\hline \hline \multirow{4}{*}{$\begin{array}{l}\text { Two-step level } \\
\text { function(2D)-solid } \\
\text { body rotation }\end{array}$} & Superbee & $-11.3710^{-10}$ & 1 & $4.4310^{-4}$ \\
\cline { 2 - 5 } & Smart & $-10.0410^{-10}$ & 1 & $5.7410^{-4}$ \\
\cline { 2 - 5 } & Koren & $-7.5110^{-10}$ & 1 & $5.7610^{-4}$ \\
\cline { 2 - 5 } & VanLeer & $-6.6310^{-10}$ & 0.99 & $6.4110^{-4}$ \\
\cline { 2 - 5 } & Van Albada & $-6.5810^{-10}$ & 0.99 & $6.6910^{-4}$ \\
\cline { 2 - 5 } & Minmod & $-4.9110^{-10}$ & 0.99 & $7.4510^{-4}$ \\
\hline \hline \multirow{4}{*}{$\begin{array}{l}\text { Gaussian } \\
\text { distribution-solid } \\
\text { body rotation }\end{array}$} & Superbee & $-9.5010^{-10}$ & 0.84 & $9.1810^{-5}$ \\
\cline { 2 - 5 } & Smart & $-13.110^{-10}$ & 0.75 & $9.6710^{-5}$ \\
\cline { 2 - 5 } & Koren & $-7.9010^{-10}$ & 0.72 & $10.310^{-5}$ \\
\cline { 2 - 5 } & VanLeer & $-5.3210^{-10}$ & 0.63 & $15.510^{-5}$ \\
\cline { 2 - 5 } & Van Albada & $-5.3210^{-10}$ & 0.63 & $19.310^{-5}$ \\
\cline { 2 - 5 } & Minmod & $-3.1410^{-10}$ & 0.45 & $25.910^{-5}$ \\
\hline \hline \multirow{3}{*}{$\begin{array}{l}\text { Gaussian } \\
\text { Distribution Lamb- }\end{array}$} & Superbee & $-8.4110^{-10}$ & 0.94 & - \\
\cline { 2 - 5 } $\begin{array}{l}\text { Oseen vortex } \\
\text { velocity } \\
\text { field }\end{array}$ & Smart & $-14.0210^{-10}$ & 0.88 & - \\
\cline { 2 - 5 } & Koren & $-8.1610^{-10}$ & 0.86 & - \\
\cline { 2 - 5 } & VanLeer & $-8.7410^{-10}$ & 0.81 & - \\
\cline { 2 - 5 } & Van Albada & $-3.8510^{-10}$ & 0.76 & - \\
\cline { 2 - 5 } & Minmod & $-3.0210^{-10}$ & 0.69 & - \\
\hline
\end{tabular}

Table 1 Sum up of the test cases

With the use of TVD schemes, numerical oscillations seem to have disappeared and all the schemes well maintain the positivity of the passive scalar. An interesting criteria is the diffusion of the maximum value (Figure 1). Here, Superbee scheme shows a weaker diffusion. RMS error gives an important clue of how the schemes resolve sharp gradients (Figure 2) and Smart and Koren limiters show a good accuracy. Among this schemes, Minmod limiter is the most diffusive.

Finally, Superbee scheme seems to give the highest level of accuracy and has been used for the discretization of the convection term in the passive scalar convection equation.

\section{Application: Plume-vortex interaction}

\subsection{Condensation trail formation}

Wing-tip vortices interact with atmosphere and exhaust jet and consequently may change substantially the fluid properties. The water saturation ratio is used as a qualitative indicator of where, in the wake flow, contrail formation is likely to begin. The saturation ratio is defined as the ratio between the actual vapor pressure and the saturation vapor pressure (or equilibrium vapor pressure at the same temperature). Contrail formation occurs when the saturation ratio is greater or equal to 1 or, in other terms, when the local relative humidity is larger than $100 \%$.

An expression for the local saturation can be given as a function of tracer concentration $Z$, including atmospheric values of static pressure and temperature. This is computed by the convection-diffusion equation (see section 2.1).

\subsection{Initial conditions}

This work is performed for a large transport aircraft: a two-engine Airbus A-330. The aircraft produces a vortex wake composed of two rolled-up vortices of opposite circulation $\Gamma_{0}=540 \mathrm{~m}^{2} \mathrm{~s}^{-1}$ and spacing $b=47.5 \mathrm{~m}$ (see Figure 3). In order to maintain the vortex system in fixed frame of reference, the opposite value of the 
descent velocity, $-\Gamma_{0} / 2 \pi b$, is imposed in vertical direction $(z)$.

In laminar case, the Crow instability must be initiated. Thus, symmetric displacements are imposed to each vortex. They are confined to fixed plane at 48 degrees with respect to horizontal. Furthermore, the 2 vortex centerlines follow a sinusoidal distribution of $1 \%$ amplitude.

The initial velocity field $v_{\theta}$ is then represented by the superposition of two Lamb-Oseen vortices. The kinematics condition (i.e. the linear addition of the two flow fields) is not quite exact, the solution of the Navier-Stokes equations for a single viscous core vortex dos not suppose the presence of a second one. However it is a reasonable approximation even if the viscous flow fields do not satisfy the Laplace's equation, because the distance between centers is large compared to the viscous core radius $r_{c}$ (with $b / r_{c}=16$ ) The initial mean pressure is obtained by integrating the radial momentum equation such that:

$$
\frac{\partial p}{\partial r}=\rho \frac{v_{\theta}^{2}}{r} .
$$

An integral model (Jacquin and Garnier, 1996) is used to describe the exhaust jet evolution extending from the exit nozzle of a CFM-56 engine until about $4 \mathrm{~s}$ the wake is generated. It provides initial plume locations with respect to vortex centers and plume diameter. At $4 \mathrm{~s}$ wake age, these values are approximately $13 \mathrm{~m}$ and $5 \mathrm{~m}$, respectively. The initial conditions for the two jet exhausts is a superposition of 2 Gaussian distributions of the passive scalar (or tracer concentration) $Z$. There is not any axial velocity centered in the two Gaussian spots.

The flight conditions are finally given by the AEROCONTRAIL European project (Mirabel et al., 1998).

\subsection{Computational domain}

Figure 3 shows the computational domain and relative position of vortex and exhaust plume centers. The numerical simulation is performed on a grid of $161 \times 81$ points in the cross plane extending from $-120 r_{c}$ to $120 r_{c}$ in the cross-stream direction (x) and from $-40 r_{c}$ to $40 r_{c}$ in the vertical direction (z). The number of points in the streamwise direction (y) is 61 . The grid is uniform in the domain defined by $-12 r_{c}<x<+12 r_{c}$ and $-4 r_{c}<z<+4 r_{c}$ with $\Delta \mathrm{x}, \mathrm{z}=0.1$. Outside, the fine grid is monotonically stretched.

The axial computational domain is related to the unstable long wave of Crow and has a length of 8.6 times the vortex separation. Periodic boundary conditions are imposed in the streamwise direction and nonreflecting boundary conditions (Thompson, 1987) are applied in the cross section. The time step is $\Delta t=0.01$ and the stability condition of the scheme is $C F L=0.4$. The simulation is performed on a Cray C 90 computer.

\subsection{Results and discussion}

Figure $4 \mathrm{a}-\mathrm{b}$ shows two-dimensional projection of the tracer concentration in an horizontal plane, at $4 \mathrm{~s}$ and $57 \mathrm{~s}$ plume age. Note that in our simulations, the engine tracer concentration represents, in general, the dynamics of the exhaust plume. However, when the engine jet is completely trapped into the vortex core, the jet tracer concentration provides a reliable signature of where the trailing vortex pair is likely to begin to touch each others (i.e. the linking process). At $57 \mathrm{~s}$, this seems to appear at approximately $y / r_{c}=90$, as seen in Figure $4 \mathrm{~b}$. It is also noticed that the characteristic time scale for the wake to descend to one vortex separation distance is defined by $T_{w}=2 \pi \frac{b^{2}}{\Gamma_{0}}$. With the aircraft parameters, $T_{w}$ is of the order of $26 \mathrm{~s}$. In these conditions, the linking phenomenon appears at approximately 2 times the characteristic time scale of the wake (i.e. at approximately 52s). The numerical results provided by Lewellen and Lewellen, 1996, give the same order of magnitude. However, the Lewellen's analysis, involving a LES Navier-Stokes approach, captures the break-up process which takes place just after the vortex linking. Detailed modeling of this process is beyond the scope of this current work.

Next, contours of the tracer concentration are discussed. Initially, Figure $4 \mathrm{a}$ shows an uniform 
distribution of a tracer, along the two engine jets. Thereafter, at 57s plume age, the exhaust plumes are wholly trapped into the vortex cores and the tracer concentration presents a maximum in the area where the linking of vortex pair occurred (i.e. at $y / r_{c}=90$ ). It is a consequence of the sinusoidal amplification of trailing vortices. According to this observation, it can be assumed that the entrainment process is reduced where the separation of vortex tubes is smaller, compared to the domain of the vortex tubes where the separation is maximum. Hence, the exchange between vortex system and ambient atmosphere is reduced, compared to the situation where the vortex tube separation is greater (i.e. at $y / r_{c}=40$ ). Finally, the plume accumulation around the vortex cores appears to be more important than in the simulation where the vortex pair remains parallel.

From Figure 5, the influence of the Crow instability on the time evolution of the maximum tracer concentration can be seen. Comparison shows that the action of the Crow instability make the dilution of the exhaust plume less efficient than in stable vortices. Before $14 \mathrm{~s}$ plume age, the profiles of the tracer concentration are quite similar and the results are of the same order of magnitude, for the calculations with and without the effect of the instability. In the near field regime, the mixing and entrainment processes appear to be dominated by the deflection and stretching of the interface between exhaust plume and ambient air. Thereafter, the plume dispersion seems to be dominated by the development of the Crow instability. As a consequence, at 40 s plume age, the tracer concentration is two times greater than this calculated without the effect of the Crow instability.

In order to characterize the rate of mixing, Figure 6 depicts time evolution of the "mixedness" $f$ (described in a previous section). Mixedness increases linearly with plume age (up to 14s) and its rate of change becomes weaker with increasing time. The evolution of this parameter as a function of aircraft wake age, presents the same behavior in the two numerical simulations (i.e. computed with an without the effect of Crow instability). Hence, it is apparent that the rate of mixing appears insensitive to the instability. However, Figure 6 shows that the only effect of the Crow instability on mixedness is a shift in its value with the same rate of growth.

In addition to test the accuracy of the tracer concentration evolution, computed throughout the vortex wake, available experimental data have been obtained by Schumann et al, (1995) (see Figure 7). The extrapolation of our modeling results (black circles) compared to the experimental data seems to be acceptable. For comparison, results of a different analysis carried out by Lewellen and Lewellen, (1997) have been plotted (black squares in Figure 7). A good agreement is found in the vortex regime, just before the development of the break-up phenomenon, at approximately 60s. Our approach does not take into account this phenomenon.

In order to know where the condensation trail occurred, Figure 8a-c shows the evolution with time of the cross flow distribution of saturation. Condensation process occurs where the saturation ratio is greater than 1. Initially, at $4 \mathrm{~s}$ the exhaust jets are supersaturated with respect to liquid water. Figure $8 \mathrm{~b}$ and $8 \mathrm{c}$ show the general features of the distortion and stretching of the exhaust plumes. During this period, a tongue of supersaturated fluid from the exhaust plume penetrated towards the vortex cores. Thereafter, the vortices descend under mutual interaction and the two exhaust plumes are trapped around the vortex cores (Figure 8c). Finally, saturation ratio profile seems to represent an actual signature of the streamlines of the supersaturated jet flow, which can be qualitatively compared to a contrail photograph (Toon et al, 1998) shown in Figure 9. In this last case, the persistence of contrail is observed in absence of shearing wind and atmospheric turbulence. So, the distribution of the plume saturation shows a ring shape centered on vortex core, as computed in Figure 8c.

\section{Concluding remarks}

MSOU scheme for the convection term of the passive scalar equation were investigated, with special attention paid to the behavior of steep gradient zones in rotational velocity fields. In this case Superbee flux 
limiter seems to damp spurious oscillation with acceptable numerical diffusion.

In second part, a three dimensional direct numerical simulation has been performed to analyze mixing and entrainment processes when vortex wake of a large two-engine aircraft undergoes Crow instability. The calculations of the time evolution of a tracer concentration have been compared with the results provided by a Large Eddy Simulation (Lewellen and Lewellen, 1997). Comparison of the models with the Schumann's data gives a good confidence in the present approach.

The mixing and entrainment processes are modified by the development of the Crow instability. According to the tracer concentration calculations, the dilution of the exhaust plume is less efficient when the flow undergone the perturbation. This is due to the reduction of the exchange between vortex system and ambient air, during the linking of the vortex pair.

The measures of molecular diffusion, described by the mixedness, varies linearly with plume age, up to 14s. This can be explained by the augmentation of the contact area between the exhaust plume and the ambient medium during the deflection and shearing regimes (from $4 \mathrm{~s}$ up to 14s). Later, the rate of change of the mixedness becomes weaker. Finally, in these early phases, the rate of mixing appears insensitive to the development of the Crow instability.

The calculations of the saturation distributions throughout the vortex wake provided a qualitative indicator where condensation phenomenon occurred in the exhaust plume and show how the ring shape is obtained in an aircraft contrail. 


\section{References}

Anderson M.R., Miake-Lye R.C., Brown R.C., and Kolb C.E., Numerical modeling of reacting engine plumes and aircraft wake interactions, AIAA 96-0948, 1996.

Boris J.P. and Book D.L., Flux corrected transport III-minimal error FCT algorithms, J. Comp. Phys., 20, 397-432, 1976.

Cetegen B.M. and Mohamad N., Experiments on liquid mixing and reaction in a vortex, J. Fluid Mech., 249, 391-414, 1993.

Crow S.C. and Bate E.R., Life span of trailing vortices in a turbulent atmosphere, J. Aircraft, 13, 476-482, 1975.

Fortuin J.P.F., Van Dorland R., Wauben W.M.F. and Kelder H., Greenhouse effects of aircraft emissions as calculated by a radiative transfert model, Annales Geophysicae, 13, 413-418, 1995.

Garnier F., Baudoin C., Woods P., Louisnard N., Engine emission alteration in the near field of an aircraft, Atmospheric Environment, 31, 12, 1767-1781, 1997a.

Garnier, F., Brunet, S. and Jacquin, L., Modeling exhaust plume mixing in the near field of an aircraft, Annales Geophysicae, 15, 1468-1477, 1997b.

Harten A., On a class of high resolution total-variation-stable-finite-difference schemes, SIAM J. Numer. Anal., 21, 1, February 1984.

Impact de la flotte aérienne sur l'environnement atmosphérique et le climat, French Academy of Sciences, Paris, 40, (1997).

Jacquin L. and Garnier F., On the dynamics of engine jets behind a transport aircraft, Conference proceedings, AGARD-584 FDP Symposium, The characterisation \& modification of wakes from lifting vehicles in fluids, 1996.

Kärcher B., A trajectory box model for aircraft exhaust plumes, J. Geophys. Res., 100, 18835-18844, 1995.

Lele, S.K., Compact finite difference scheme with spectral-like resolution, J. Comp. Phys., 03, 6-42, 1992.

Lewellen, D.C. and Lewellen, W.S., Large-eddy simulations of the vortex-pair breakup in aircraft wakes, AIAA Journal, 34, 11, 2337-2345, 1996.

Lowery P.S. and Reynolds W.C., Numerical simulation of a spacially developping forced plane mixing layer, Report NTF-26 Stanford University, 1986.

Mirabel, P., Garnier, F., Gayet, J.F., Sussmann, R., Ström, J., Wendling, P., Formation processes and radiative properties of particles in aircraft wakes: AEROCONTRAIL, $R \& D$ program environment and climate, Report N ENV4-CT95-0157, 1998

Ponater, M., S. Brinkop, R. Sausen, and U. Schumann, Simulating the global atmospheric response to 
aircraft water vapor emissions and contrails: a first approach using a GCM, Annales Geophysicae, 14, 941960, 1996.

Schumann U., Konopka P., Baumann R., Busen R., Gerz T., Schlager H., Schulte P. and Volkert H., Estimate of diffusion parameters of aircraft exhaust plulmes near the tropopause from nitric oxyde and turbulence measurements, J. Geophys. Res., 100, 14147-14161, 1995.

Thompson, K.W., Time dependant boundary conditions for hyperbolic systems , J. Comp. Phys., 89, 439461, 1990.

Toon O.B., Myake-Lye R.C., Subsonic Aircraft: Contrail and Cloud Effects Special Study (SUCCESS), Geophys. Res. Lett., 25, 8, 1109, 1998.

Waterson, N.P., and Deconinck, H, A unified approach to the design and application of bounded high-order convection schemes, von Karman Institute for Fluid Dynamics, 1995.

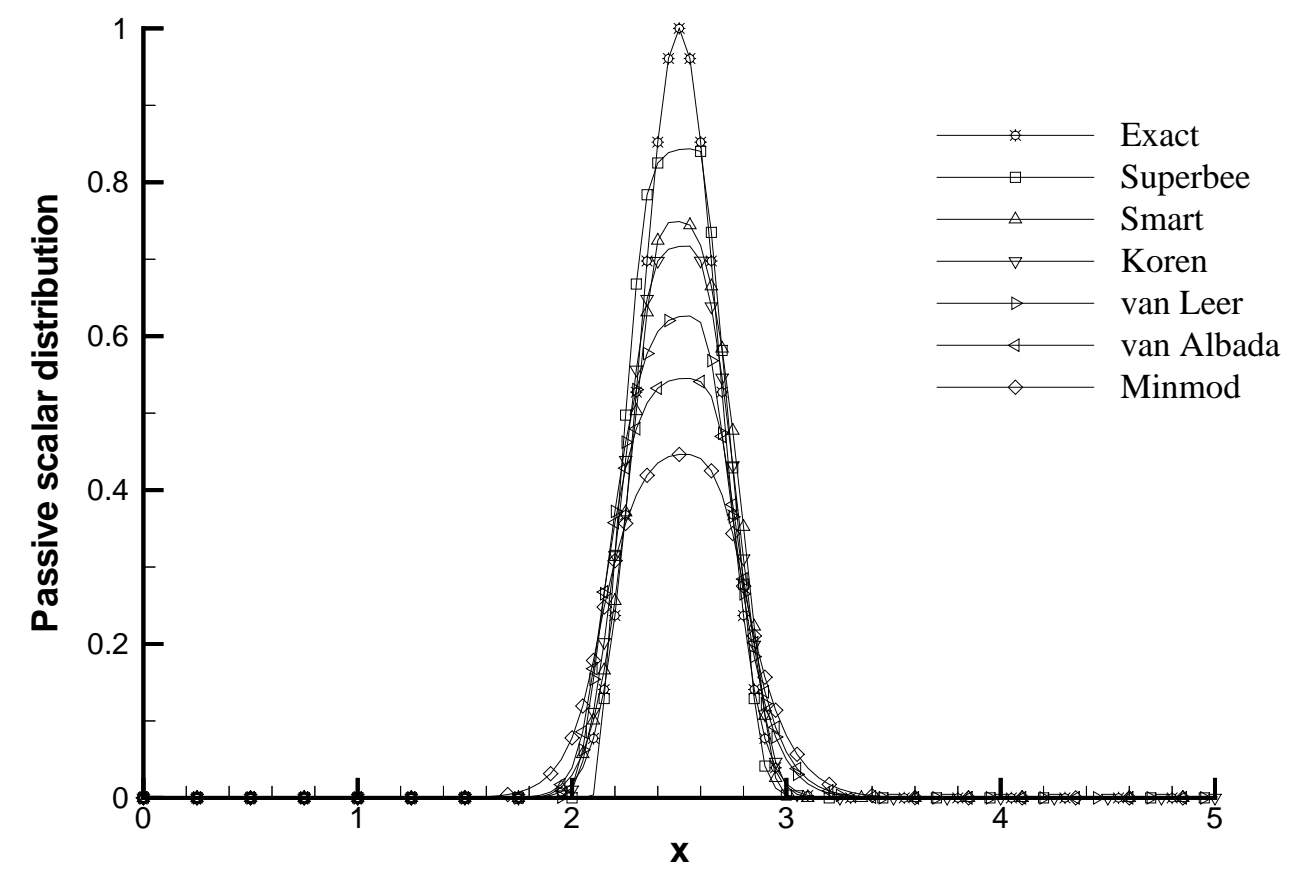

Figure 1 : Gaussian case in solid-body rotation. Numerical diffusion of 6 flux-limiters (outlet profiles) 


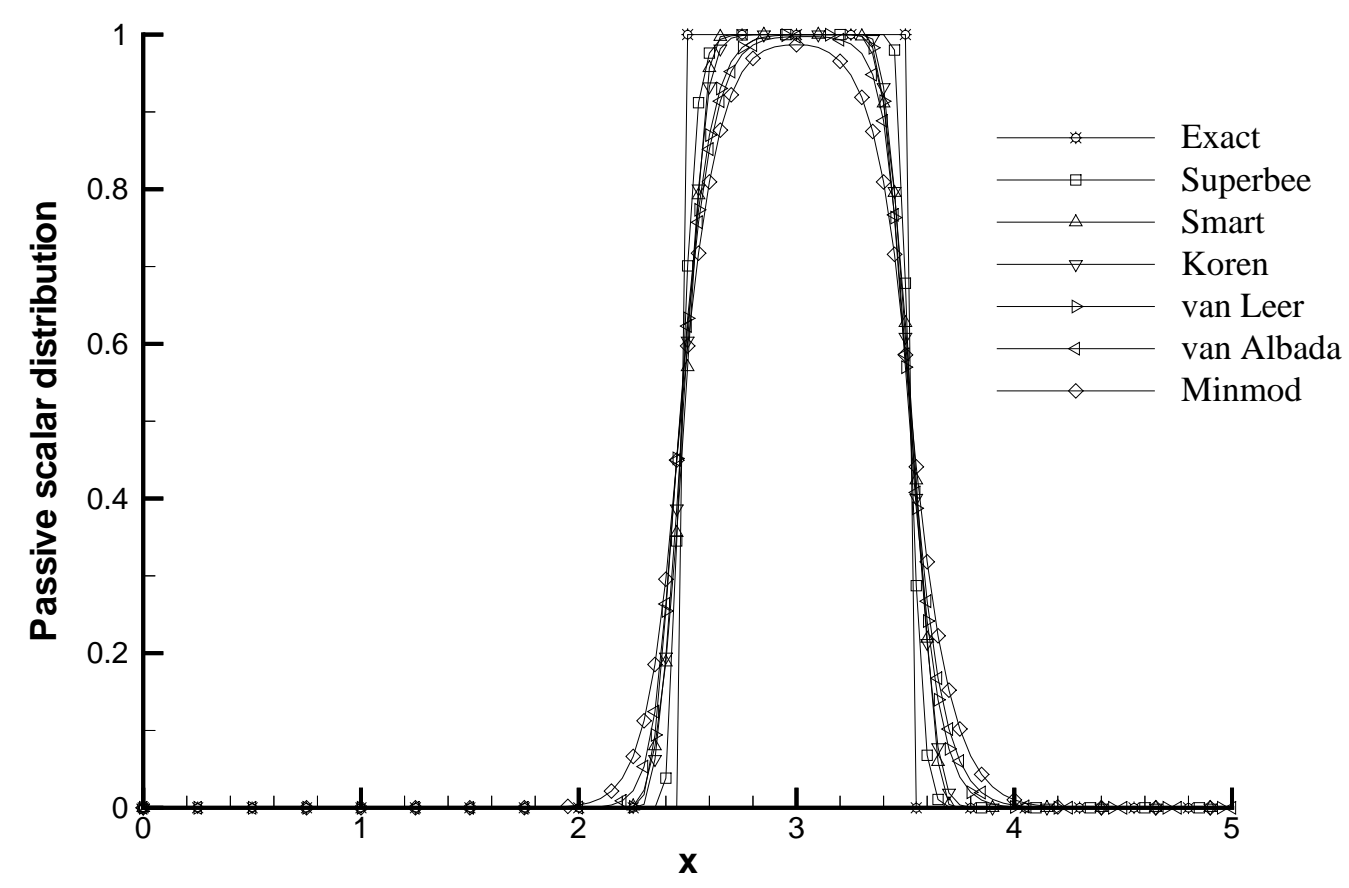

Figure 2 : Two-level step case in solid-body rotation. Numerical diffusion of 6 flux-limiters (outlet profiles)

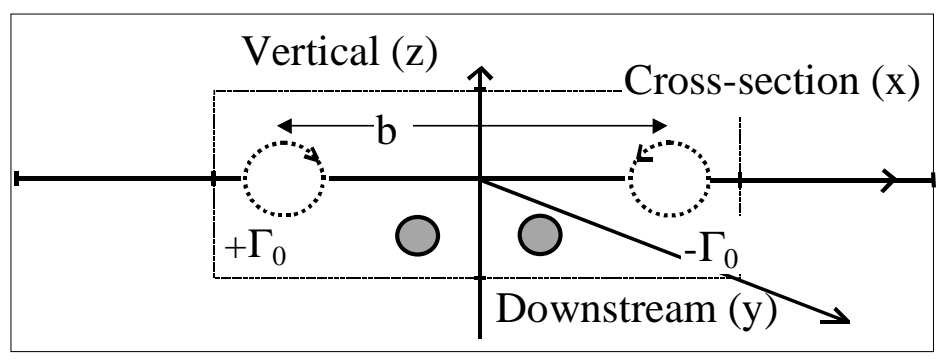

Figure 3 : Computational domain and relative position of the vortex pair (dot line) and passive scalar spots (solid line) 


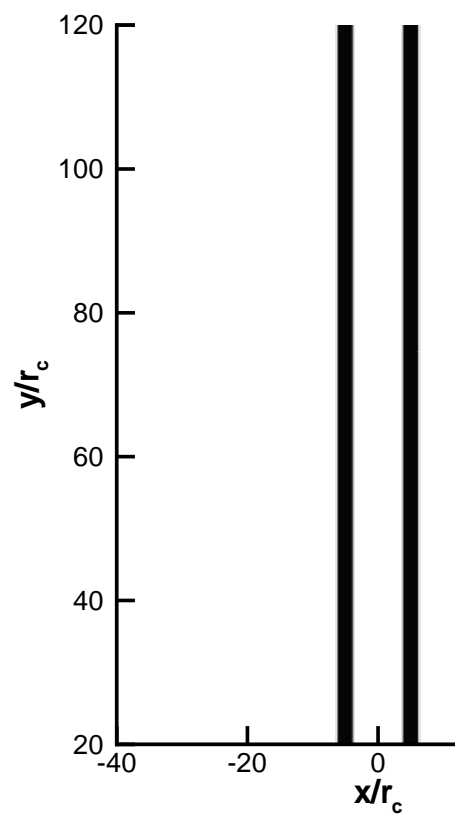

a

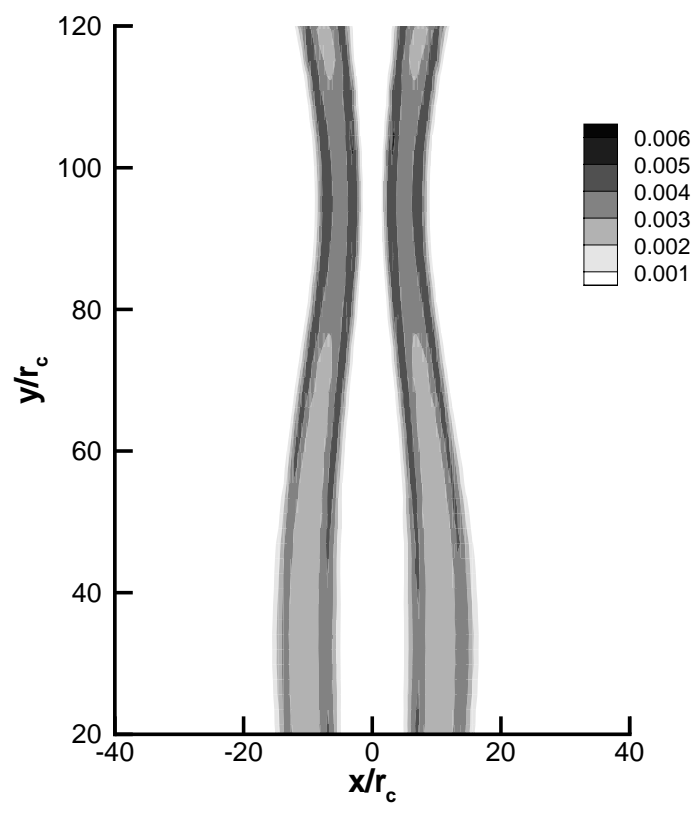

b

Figure 4 : 2-D vertical projection of the tracer concentration for a twin engine A-330 Airbus. (a) initial condition; (b) plume age 57s. These results are computed from a three-dimensional Direct Numerical Simulation.

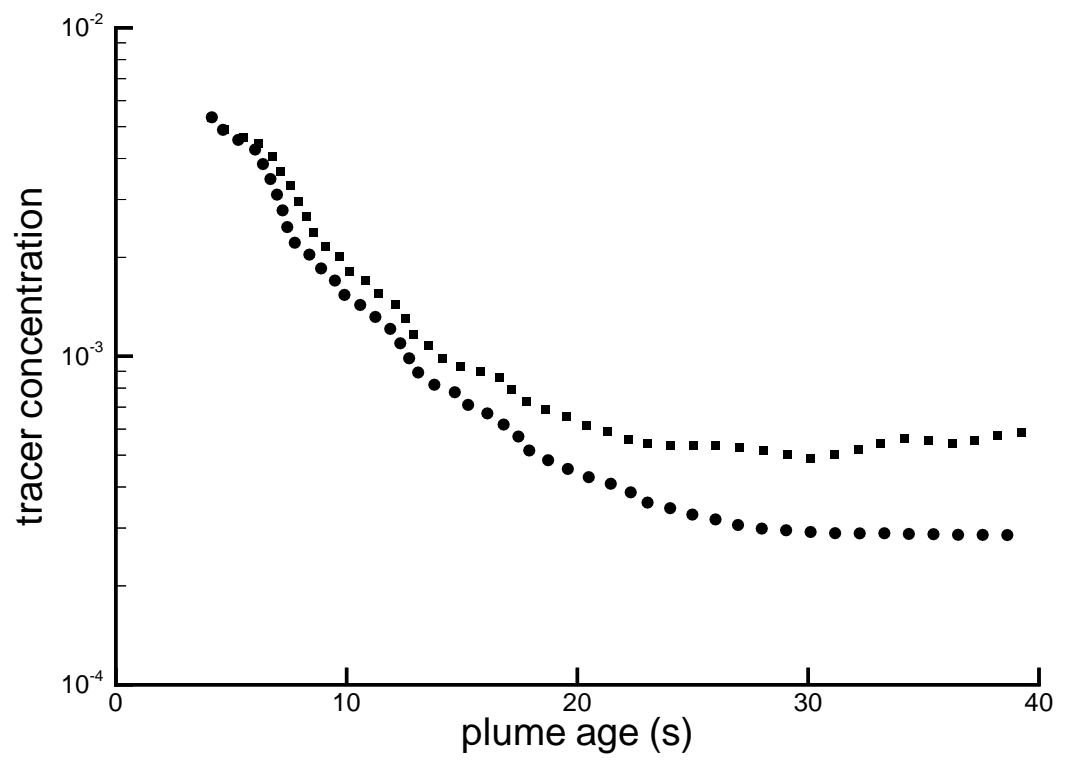

Figure 5 : Evolution of the tracer concentration as a function of

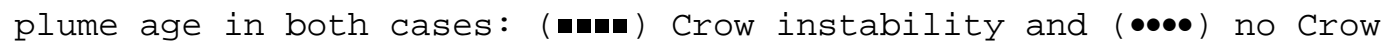
instability 


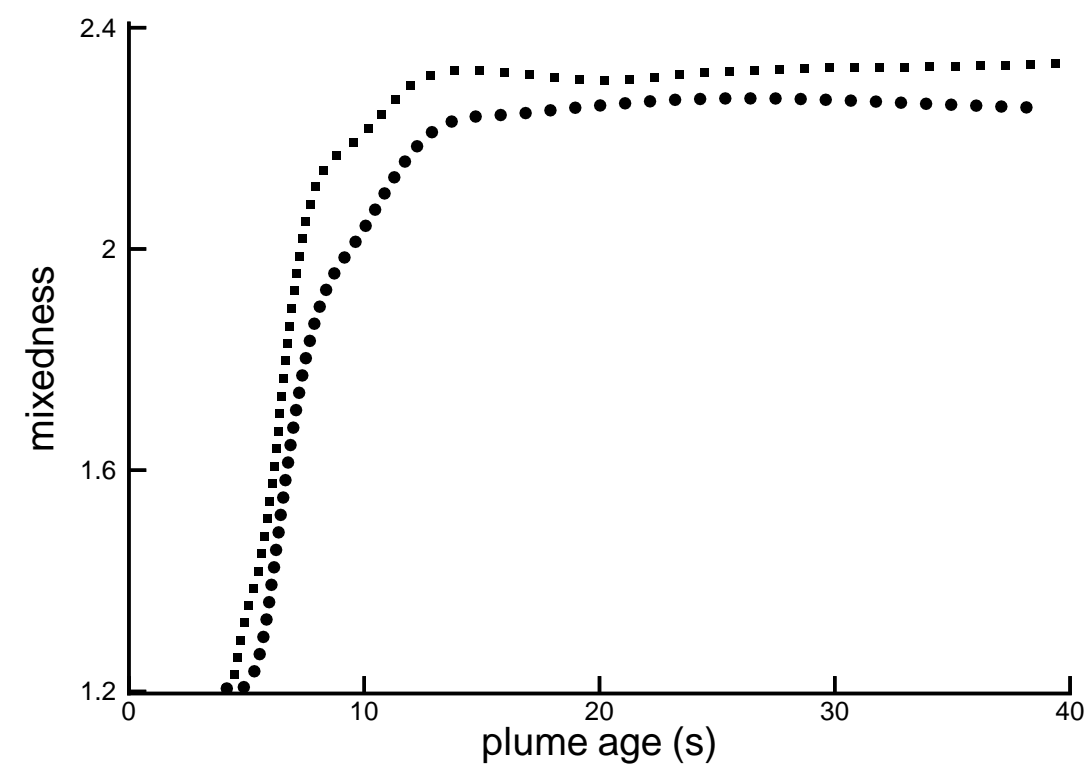

Figure 6 : Time evolution of the mixedness plots comparing with the Crow instability (-ma) and without Crow instability $(\bullet \bullet \bullet \bullet)$

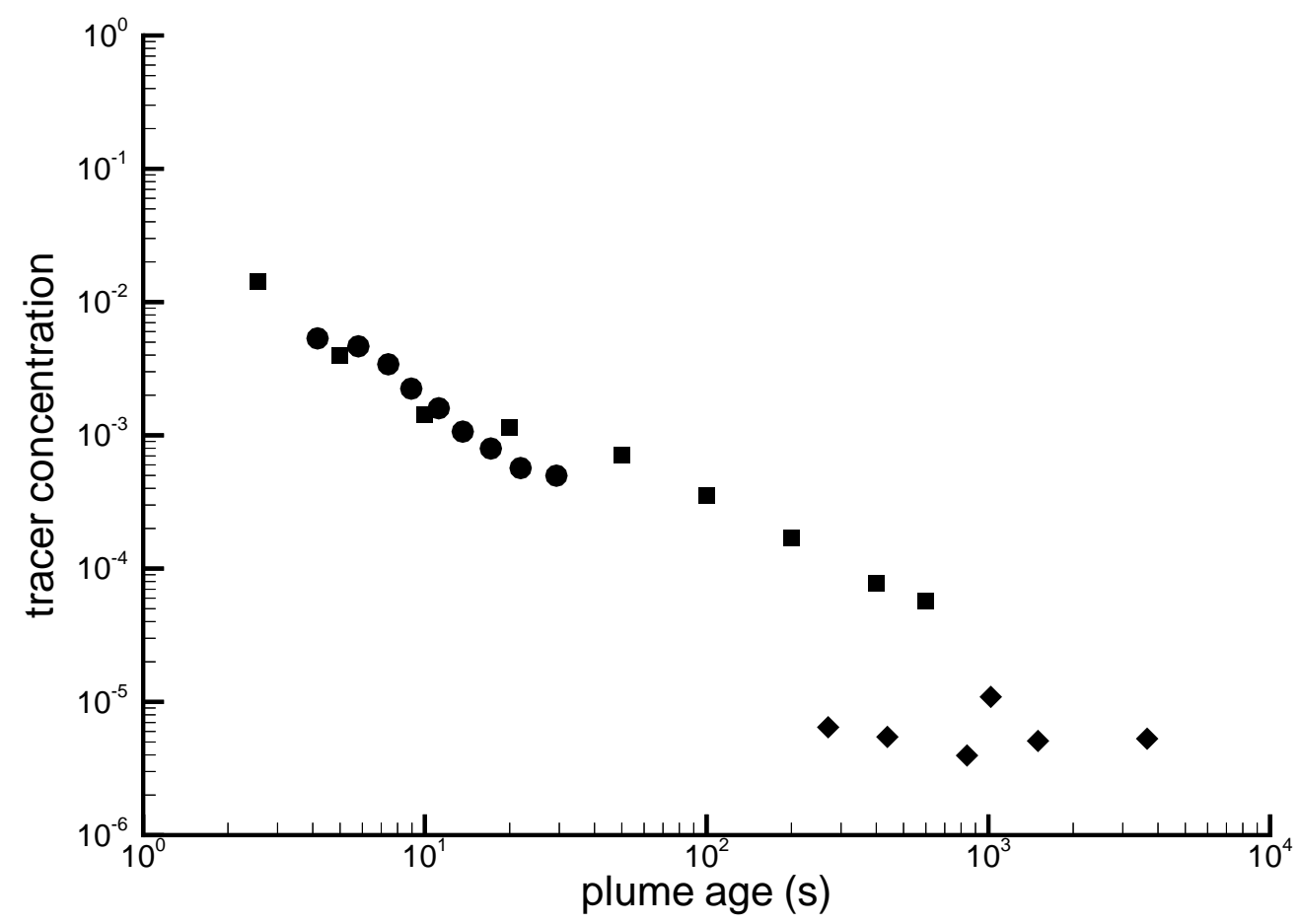

Figure 7 : Maximum tracer concentration plots of exhaust plume of a large transport aircraft. Comparison of

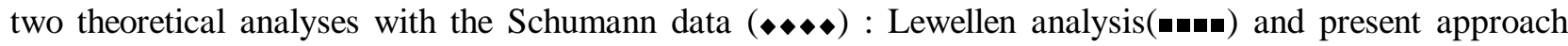
$(\bullet \bullet \bullet \bullet)$ 
a

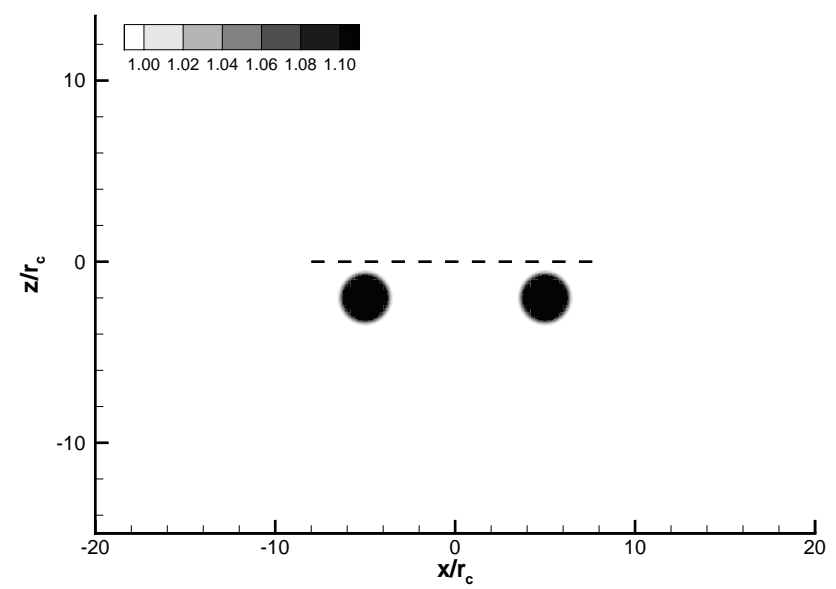

$\mathrm{b}$

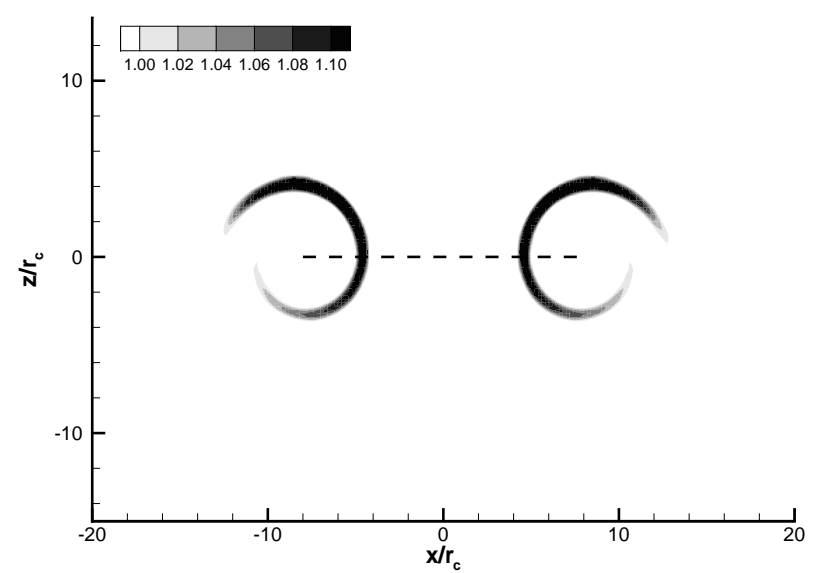

c

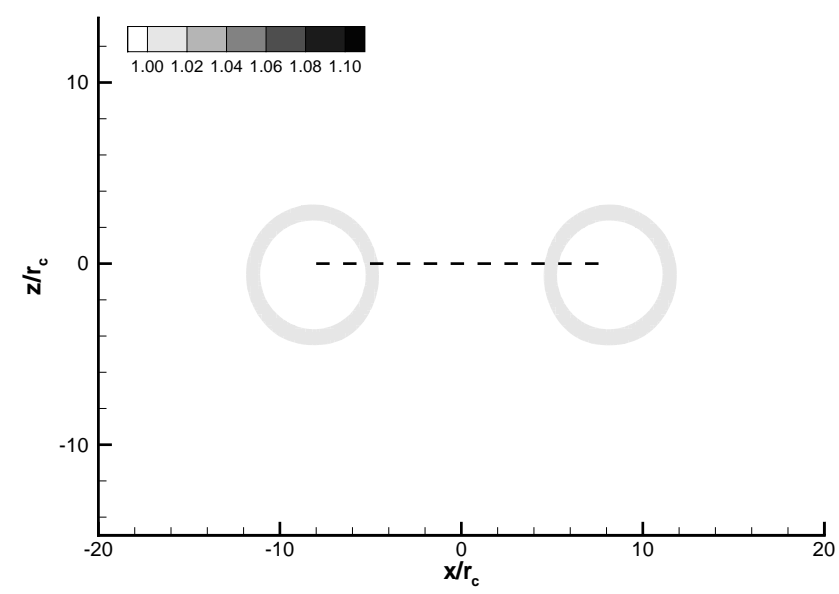

Figure 8 : Cross-section of the saturation ratio distribution. (a) initial condition; (b) plume age 14s; (c) plume age $41 \mathrm{~s}$. Values greater than 1 indicate condensation occurrence. The vortex separation distance $b$ is drawn in dashed line. 
Figure 9 : Photograph of an aircraft contrail observed in absence of shearing wind and atmospheric turbulence, during SUCCESS experiment (Toon et al, 1998). 